Joscha Wullweber

\title{
Hegemoniale Strategien: Das Ringen um Akzeptanz in der politischen Governance der Nanotechnologie
}

\section{Einleitung}

Seit gut zehn Jahren wird von Nanotechnologie als der Zukunftstechnologie des 21. Jahrhunderts gesprochen. Der Begriff Nanotechnologie (nano von griechisch »Zwerg «) steht dabei für Technologie der Miniaturisierung - ein Nanometer entspricht $0,000000001 \mathrm{~m}$. Der Begriff ist jedoch äußerst indifferent, handelt es sich doch eher um ein technologiepolitisches Projekt als um konkrete Techniken oder Technologien. ${ }^{1}$ Große Summen werden in diesen Bereich investiert, und fast jedes Industrie- und Schwellenland hat inzwischen seine eigenen Nanotechnologie-Programme entwickelt. Wöchentlich kommen neue »Nano «-Produkte auf den Markt.

Kritik an der Nanotechnologie gibt es bereits seit einigen Jahren. Akteure aus der Zivilgesellschaft, wie Gewerkschaften, Umwelt- und Verbraucherverbände, aber auch Kirchen und Rückversicherer, äußern ernstzunehmende Bedenken, und viele Studien warnen vor möglichen Gefahren zum Beispiel von Nano-Partikeln für Mensch und Umwelt. Erstaunlicherweise gibt es trotz dieser Kritik kaum eine Organisation in der Zivilgesellschaft, die die Nanotechnologie gänzlich ablehnt. Im Gegenteil sehen die meisten am Diskurs um die Nanotechnologie beteiligten Akteure vor allem die Chancen dieser Technologieentwicklungen. Der Grund hierfür, so die zentrale These dieses Beitrags, ist in der - im Sinne der Nanotechnologie-Protagonisten - erfolgreichen politischen »Governance« der Nanotechnologie zu suchen.

\section{Das Ringen um gesellschaftliche Akzeptanz}

Im Folgenden wird die Governance der Nanotechnologie an einigen zentralen Beispielen aufgezeigt. Hierbei wird ein hegemonietheoretisch gewendeter GovernanceBegriff verwendet, der die prekäre, kontingente und diffuse Dimension strategischer »Lenkung " hervorheben soll. ${ }^{2}$ Eine solche Herangehensweise beinhaltet zu untersuchen, wie durch Governance gesellschaftliche und politische Verhältnisse in einem interessengeleiteten Prozess verändert werden. Bezogen auf die politische Governance der Nanotechnologie, geht es in diesem Beitrag darum, wie innerhalb

1 Vgl. Wullweber 2008; 2010.

2 Ähnlich bezeichnen Hajer und Wagenaar (2003, S. 27) Governance als Prozess, in dem "people and communities ... struggle with concrete issues in settings that are rife with conflict and power differentials «. 
des Nanotechnologie-Diskurses um gesellschaftliche Akzeptanz gerungen und ein bestimmter Wahrheits- und Handlungshorizont generiert wird. ${ }^{3}$

Politik wird in diesem Beitrag daher als Ringen um gesellschaftliche Stabilisierungen, als Ringen konkurrierender Artikulationen um die Instituierung gesellschaftlicher Ordnungen, verstanden. Der Begriff der Artikulation wird hier verwendet, um das In-Beziehung-Setzen von Handlungen, Ereignissen, Identitäten und Dingen zu beschreiben, aus der eine bestimmte gesellschaftliche Bedeutung generiert wird. In dem stets konflikthaften Ringen zwischen verschiedenen Artikulationen in der Öffentlichkeit zeichnet sich der Versuch ab, die Beschreibung gesellschaftlich anerkannter Realität zu dominieren.

Hegemoniale Auseinandersetzungen stellen demnach ein grundlegendes gesellschaftliches Organisationsprinzip von demokratischen Gesellschaften dar. ${ }^{4}$ Diese Konzeptualisierung von Hegemonie beinhaltet eine Auffassung von Macht, die vor allem auf der Fähigkeit beruht, die Interessen einer bestimmten gesellschaftlichen Gruppe als unter anderem politische, kulturelle und sozioökonomische Struktur zu universalisieren. Die Kongruenz von Interessen kann darüber erreicht werden, dass die Interessen verschiedener Gruppen bereits in der Initiierungsphase von Institutionen oder politischen Projekten beachtet und bis zu einem gewissen Grad integriert werden.

Zur Erlangung von Hegemonie gibt es nach Gramsci ${ }^{5}$ zwei allgemeine Strategien, den Transformismus und die expansive Hegemonie. Erstere beschreibt eine eher defensive Vorgehensweise, in welcher antagonistische politische Kräfte beispielsweise durch Kooptation neutralisiert werden. Durch diese Art der Strategie kann allerdings nur ein passiver Konsens der Massen erlangt werden. Die konkurrierenden gesellschaftlichen Kräfte werden nicht überzeugt, sondern politisch desorganisiert. Zur Erlangung eines national-populären Kollektivwillens - eines aktiven Konsenses der Massen - bedarf es nach Gramsci einer Strategie der expansiven Hegemonie.

Laclau und Mouffe betonen in ihrer grundlegenden Schrift von 1985 die Bedeutung von hegemonialen Diskursen für die Konstituierung eines politischen Feldes und für die Konstruktion von Subjekten. Ein hegemonialer Diskurs ist demzufolge eine in sich differenzierte, aus sozialen Praktiken entstandene gesellschaftlich vorherrschende Realität, die zur Stabilisierung eines bestimmten Handlungs- und Wahrheitshorizonts führt. Diskurse sind demnach kein bloßes Abbild der Realität, sondern stellen diese zugleich permanent her. Gesellschaft und diskursives Feld sind identisch. Hegemonie ist für Laclau und Mouffe dementsprechend »das Schlüsselkonzept, um Politik zu denken «, ${ }^{6}$ und beinhaltet das strategische Ringen um Wahrheit. Ein Wahrheitshorizont setzt voraus, dass die gesellschaftlich produzierte

3 Canzler und Marz (2011) verwenden einen Valorisierungsansatz, um die Etablierung der Wasserstoff- und Brennstoffzellentechnologien in Deutschland zu beschreiben.

4 Vgl. Gramsci 1991; Laclau, Mouffe 1985.

5 Vgl. Gramsci 1971, S. 55 ff., 106 ff., 129 ff.

6 Laclau 1998, S. 277.

Leviathan, 40. Jg., 1/2012 
Wahrheit zumindest von einem Teil der Menschen auch als wahr angesehen wird. Bei dem Versuch der Durchsetzung von bestimmten Bedeutungskonstruktionen handelt es sich daher auch um »einen Kampf um die Objektivität «. ${ }^{7}$

Hegemoniale Strategien können demnach als soziale Handlungen konzeptualisiert werden, die ein spezifisches hegemoniales Projekt bzw. einen gesellschaftlichen Diskurs voranzutreiben und zu stabilisieren bestrebt sind. Es gibt in der Regel nicht die eine Strategie und das Interesse einer bestimmten Gruppe, die ein Projekt protegieren. Vielmehr artikulieren sich innerhalb eines hegemonialen Projekts zumeist verschiedene Interessen und Strategien, die durchaus in sich Widersprüche zum Ausdruck bringen und produzieren können. Auch können bestimmte, dem Projekt zugrunde liegende Diskurse eine gewisse »Eigendynamik « entfalten, da diese unter Umständen auf unerwartete und ungeplante Weise von einer Vielzahl von Akteuren aufgegriffen, in anderen Kontexten artikuliert und damit insgesamt verschoben oder verzerrt werden. Hegemoniale Projekte sind daher nur bedingt steuerbar. Das bedeutet auch, dass jedes hegemoniale Projekt durch seinen spezifischen Kontext geprägt und auf besondere Weise fortgeschrieben wird.

\section{Strategien im Diskurs um die Nanotechnologie}

Vielen Protagonisten der Nanotechnologie ist die Bedeutung der gesellschaftlichen Akzeptanz bewusst, insbesondere denjenigen, die bereits in den Gentechnik-Diskurs involviert waren: »Vertrauen in die Nanotechnologie und deren Akzeptanz durch die Allgemeinheit sind für ihre langfristige Entwicklung und die Nutzung ihrer potenziellen Vorteile entscheidend $\iota^{8}$ Denn vor allem in Europa mussten GentechnikBefürworterInnen in den 1990er Jahren die Erfahrung machen, dass diese Technologie nicht einfach (»von oben «) durchgesetzt werden konnte und eine neutrale bis tendenziell positive Grundhaltung der Bevölkerung in anhaltende Ablehnung umschlug. Aus diesem Grund warnt die Europäische Kommission, dass der Nanotechnologie ohne ernsthafte Kommunikationsbemühungen eine "ungerechtfertigte " schlechte öffentliche Stimmung ähnlich wie bei der Gentechnik entgegenschlagen könnte. ${ }^{9}$ Die meisten Menschen können mit dem Begriff Nanotechnologie immer noch relativ wenig anfangen. Zugleich herrscht eine eher positive Grundhaltung in der Öffentlichkeit gegenüber der Nanotechnologie vor. Das Konfliktpotenzial scheint also gering. Allerdings ist auch von anderen Technologien bekannt, dass diese von der Öffentlichkeit zunächst positiv aufgenommen wurden, insbesondere wenn große Hoffnungen auf ökonomische und gesundheitliche Potenziale bestanden. Erfahrungsgemäß ist also die Begeisterung für technologische Entwicklungen zu Beginn relativ hoch. Auch die Gentechnik wurde anfangs eher positiv gesehen, was sich dann jedoch innerhalb eines relativ kurzen Zeitraums änderte.

7 Gramsci 1991, S. 1412.

8 Europäische Kommission 2004, S. 23.

9 Vgl. ebd. 
Der bislang eher positiven Stimmung gegenüber der Nanotechnologie fehlt noch ein robustes Fundament, das einen Stimmungsumschwung erschweren würde. Vor allem der fehlende Bekanntheitsgrad der Nanotechnologie wird von deren BefürworterInnen als Problem gewertet. ${ }^{10}$ Strategisch gilt es für die Protagonisten der Nanotechnologie vor allem zu vermeiden, dass in der Bevölkerung Angst vor der Nanotechnologie entsteht. Schlagzeilen wie »Angst vor den Nanogiften «, "Angst vor Nano im Regal « oder »Alert over ... nanotechnology Frankenfoods « oder auch "Nanotech food is ten times scarier than genetically engineered « ${ }^{11}$ sind Botschaften, deren Ausbreitung ein Problem für die Akzeptanz der Nanotechnologie darstellen würde. Für die Protagonisten der Nanotechnologie gilt es, der Verbreitung solcher Botschaften, die von der PR-Agentur Burson-Marsteller als »fear factors « bezeichnet werden, entgegenzuwirken. ${ }^{12}$

Die von zivilgesellschaftlichen Akteuren artikulierte Kritik erfordert (öffentliche) Reaktionen seitens der Protagonisten der Nanotechnologie. Denn bei dauerhaftem Nicht-Reagieren besteht die Gefahr, dass die Forderungen nach einer stärkeren Regulierung der Nanotechnologie in eine Ablehnung der Nanotechnologie überhaupt umschlagen und somit eine "antagonistische Äquivalenzkette " gebildet wird, wie es Ernesto Laclau beschreibt. ${ }^{13}$ Das könnte ein Problem für die Akzeptanz der Nanotechnologie werden, wenn sich diesen antagonistischen Forderungen entsprechend viele und bedeutende Akteure anschließen würden, so dass die gesellschaftliche Stimmung insgesamt umschlagen oder der hegemoniale Konsens unter wichtigen gesellschaftlichen Akteuren zerbrechen könnte.

Die Stimmung der Bevölkerung in den meisten Industrieländern der Nanotechnologie gegenüber bietet also eine gute Grundlage für deren Akzeptanz, kann sich aber relativ schnell zu einer negativen und ablehnenden Haltung entwickeln. Entsprechend richten sich die verschiedenen Strategien der Akteure aus. Die Reaktion auf die Kritik muss freilich als Suchprozess verstanden werden, in dem sich über konkret geführte gesellschaftliche Auseinandersetzungen eine oder mehrere mögliche Strategien durchsetzen oder sich auch keine kongruente Strategie herausbildet. ${ }^{14}$ Für die Analyse des Nanotechnologie-Diskurses wurden sich wiederholende Strategie-Muster, die »Familienähnlichkeiten « (Wittgenstein) aufweisen, analytisch zu Strategie-Clustern zusammengefasst. Ein Strategie-Cluster besteht demzufolge aus niemals identischen, aber dennoch in ähnlicher Form wiederkehrenden strategischen Handlungen.

\section{1. »Wir wollen (eigentlich) das Gleiche!"}

Eine Reaktion auf Forderungen nach einer stärkeren Regulierung der Nanotechnologie besteht etwa in dem Hinweis darauf, dass bereits an verschiedenen Formen

10 NNAP 2008, S. 34.

11 Vgl. Ross 2006; Bullis 2006; Poulter 2008; Feder 2006.

12 Burson-Marsteller AG 2004, S. 3.

13 Laclau 2005, S. 77 ff.

14 Siehe ausführlich Wullweber 2010, S. 139 ff., 233 ff.

Leviathan, 40. Jg., 1/2012 
der Regulierung gearbeitet werde. Diese Reaktion kann als Strategie-Cluster Äquivalenzierung von sozialen Praktiken bzw. Artikulationen beschrieben werden. Es wird eine Verbindung (»Äquivalenzkette «15) zwischen den Forderungen und der hegemonialen Governance der Nanotechnologie hergestellt, wodurch potenziell antagonistische Forderungen in den hegemonialen Diskurs integriert werden.

Innerhalb des Policyfeldes der Risikoregulierung werden vor allem folgende Kritikpunkte artikuliert: An keiner Stelle des gesamten Produktlebenszyklus von Nanotechnologie-Produkten gibt es bisher speziell abgestimmte und vorgeschriebene toxikologische Tests. Vielmehr werden weiterhin die bereits bestehenden unspezifischen (Risiko-)Regulierungen angewendet. Es gibt bislang keine spezifische Kennzeichnungspflicht und fast keine nationale - geschweige denn internationale - Risikoregulierung der Nanotechnologie. Es kann auch nicht annähernd von einer im nationalen oder europäischen Rahmen einheitlichen Umgangsweise mit dem Gefahrenpotenzial der Nanotechnologie gesprochen werden.

Die EU-Kommission weist nun darauf hin, dass die Forderungen nach Risikoregulierung legitim und verständlich sind. ${ }^{16}$ Vor dem Hintergrund, dass es bislang keinen rechtlichen Regulierungsrahmen für die Nanotechnologie gibt und dass von verschiedenen Akteuren darauf hingewiesen wird, dass Risiken der Nanotechnologie durch die bereits existierenden toxikologischen Tests und Regulierungen längst nicht genügend erfasst werden, sind diese Aussagen vor allem als Strategien der Abwehr zu deuten.

Nach einer Umfrage unter WissenschaftlerInnen im Bereich Nanotechnologie stellt das Scientific Committee on Emerging and Newly Identified Health Risks (SCENIHR) der EU-Kommission fest, dass ExpertInnen einhellig der Meinung sind, dass die negativen Auswirkungen von Nanopartikeln nicht von der Toxizität von makroskopischen Materialien abgeleitet werden können. Das Komitee kommt deswegen zu dem Schluss, dass die derzeitigen Risikobewertungsmethoden modifiziert werden müssen, um in der Lage zu sein, mit möglichen Gefahren der Nanotechnologie umgehen zu können. ${ }^{17}$ Forschungsbedarf wird insbesondere hinsichtlich der Ökotoxizität von Nanopartikeln gesehen, wenn diese unvermeidlich in Kontakt mit Menschen kommen (etwa Kosmetika). Auch fehle es an einer umfassenden Risikobewertung.

Staatliche Akteure und die EU-Kommission nehmen die Forderungen nach spezifischen Risiko-Regulierungen der Nanotechnologie auf und integrieren diese in den eigenen Forderungs- und Handlungskatalog. So verlangt auch die EU-Kommission zum Schutz der allgemeinen Gesundheit und Sicherheit und zum Schutz von Umwelt und Verbrauchern, dass die Sicherheitsbedenken schnellstmöglich identifiziert, die Gesundheits-, Umwelt- und Risikoaspekte in die Forschungs- \& Entwicklungs-Ak-

15 Laclau, Mouffe 1985, S. $127 \mathrm{ff}$.

16 European Commission 2007, S. 3.

17 SCENIHR 2005, S. 6. 
tivitäten (F\&E-Aktivitäten) integriert werden, die Sammlung von (öko-)toxikologischen Daten unterstützt wird und mögliche Risiken identifiziert werden. ${ }^{18}$

Das Gefahrenpotenzial der Nanotechnologie wird sehr unterschiedlich bewertet. Das Umweltbundesamt argumentiert, dass Nanopartikel in der Regel fest in Schichten oder Dispersionen eingebettet sind und nach derzeitigem Wissen daher eine Freisetzung und damit eine Gefährdung durch diese Partikel kaum zu erwarten sei. Diese Argumentation ist insofern verwunderlich, da zugleich darauf hingewiesen wird, dass bislang nur unzureichende Informationen über die Freisetzung von Nanopartikeln durch Alterungs- und Abbauprozesse vorliegen. Mögliche Gefahren werden allerdings bei deren Verwendung in Kosmetika und Bioziden sowie bei der Herstellung von Nanopartikeln selbst gesehen, da bei diesen Produkten und Verfahren eine Freisetzung von Nanopartikeln zu vermuten sei. ${ }^{19}$ In einer aktuellen Studie des Sachverständigenrats für Umweltfragen von 2011 wird allerdings auf erhebliche Risiken durch Nanotechnologie-Produkte hingewiesen.

Das Unwissen über potenzielle Gefahren im Bereich Nanotechnologie führt derzeit zu der scheinbar paradoxen Situation, dass Nanotechnologie-Produkte nicht verboten sind, sondern unreguliert - genauer: nicht spezifisch reguliert - auf den Markt kommen. Das wird damit begründet, »dass für eine realistische Abschätzung der Exposition, Gefährdung und dem einhergehenden Risiko die gegenwärtige Datenlage nicht ausreicht. Daher sind derzeit auch spezifische regulatorische Maßnahmen nicht möglich, da völlig unklar ist, worauf sie eigentlich abzielen sollten «.20 Auch die Feststellung einer Studie des Bundesministeriums für Bildung und Forschung (BMBF), dass ein streng ausgelegtes Vorsorgeprinzip wegen der enormen Wissenslücken hinsichtlich der Risiken im Bereich Nanotechnologie ein Moratorium der Nanotechnologie-F\&E zur Folge haben müsste, führt nicht zum Stopp der Forschung, sondern stattdessen zu der Feststellung, dass aus diesem Grund das Vorsorgeprinzip in diesem Bereich nur eingeschränkt angewendet werden könne. ${ }^{21}$

Nach der Integration der Forderung nach Risiko-Regulierung in die vorherrschende Governance der Nanotechnologie wird die Kritik modifiziert und angepasst. So wird die Möglichkeit eines Moratoriums von der EU-Kommission zwar erwähnt, aber sogleich wieder verworfen, indem etwa argumentiert wird, dass ein solches schwer umzusetzen sei, da sich bereits Nanotechnologie-Produkte auf dem Markt befänden und der universelle und horizontale Charakter der Nanotechnologie es schwierig mache, diese zu kontrollieren. ${ }^{22}$ Ebenso wird eine umfassende und spezifische Regulierung der Nanotechnologie als schwer umsetzbar beurteilt und nur der inkrementelle Ansatz, also die schrittweise Anpassung der bereits existierenden Regulierungen, als angemessen erachtet.

18 Vgl. Europäische Kommission 2004, S. 23 f.

19 Umweltbundesamt 2006, S. 5 f.

20 Wörle-Knirsch, Krug 2007, S. 111.

21 Vgl. Lösch et al. 2008, S. 37.

22 Vgl. European Commission 2004, S. 24.

Leviathan, 40. Jg., 1/2012 
Vom derzeit geltenden Regulierungsansatz aus wird es zu einer Überarbeitung des bestehenden Regulierungsrahmens innerhalb der EU nach der seit Juni 2007 in Kraft getretenen EU-Chemierichtlinie REACh (Registration, Evaluation, Authorisation and Restriction of Chemicals) kommen. Von zivilgesellschaftlicher Seite wird allerdings kritisiert, dass REACh zu einem Zeitpunkt verhandelt wurde, als die Risiken der Nanotechnologie noch nicht auf der Agenda standen. Das bedeutet, dass sich die aktuell geltende Richtlinie bislang noch gar nicht mit den Auswirkungen unterschiedlicher Größen der Stoffe befasst. Wenn Stoffe und Chemikalien in der herkömmlichen Form als ungefährlich eingestuft werden, gilt das bisher unabhängig von ihrer Größe. Auch wird nicht berücksichtigt, dass Nanopartikel durch Agglomeration ihre Eigenschaften verändern können. Im Übrigen müssen nur solche Materialien umfassend auf mögliche Toxizität getestet werden, die in großen Mengen hergestellt werden. Für Produktionsmengen unter 100 Tonnen gibt es bisher lediglich eine eingeschränkte Pflicht zur Evaluation, und unter einer Tonne ist die Registrierung des Stoffs nicht vorgeschrieben. Viele Nanopartikel werden aber bislang erst in geringen Mengen hergestellt und daher nicht durch REACh erfasst. Auch eine Studie des BMBF urteilt deshalb: "Gegenwärtig gilt REACh ... als ungeeignet ${ }^{23}{ }^{23}$

Als Alternative zu gesetzlich vorgeschriebenen Regulierungsformen und angesichts der Defizite der derzeit geltenden Regulierungen gibt es verschiedene Strategien, einen freiwilligen Verhaltenskodex (Code of Conduct) zu etablieren. Dieser Kodex sei nötig, da bislang ein » Mangel an wissenschaftlichen Kenntnissen und das Fehlen von Beweisen zu gesundheitlichen und sicherheitsbezogenen Risiken der Nanotechnologie ... eine Regulierung ... unmöglich « machen. ${ }^{24}$ Die Idee hierbei ist, dass die Industrie sich eigenverantwortlich Leitlinien für die Nanotechnologie-F\&E setzt und gleichzeitig auf staatliche Regulierungen weitgehend verzichtet wird. Ein solcher Regulierungsansatz wird derzeit von staatlichen Stellen und verständlicherweise auch von der Industrie favorisiert. Einige Unternehmen wie BASF, Bayer oder Degussa haben bereits eigene Verhaltenskodizes entwickelt. Allerdings sind auch innerhalb der staatlichen Administration kritische Stimmen zu hören: »Man verabschiedet sich von der öffentlichen Aufsicht durch den Rückzug auf freiwillige >Codes of Conduct « und `Best Practices von Industrie und Privatwirtschaft $« .{ }^{25}$ Die Forderung nach Risikoregulierung wird also zwar mit diesem freiwilligen Regulierungsansatz aufgenommen, gleichzeitig allerdings strategisch-selektiv auf eine solche Weise modifiziert, dass statt einer rechtlich verbindlichen Risikoregulierung ein rechtlich nicht bindender Ansatz verfolgt wird.

Aus der Unzulänglichkeit der derzeitigen Regulierungsformen, die von vielen Akteuren anerkannt wird, und dem Unwissen über mögliche Risiken folgt also kein Verbot von Nanotechnologie-Produkten. Während auf Forderungen nach Regulierung der Nanotechnologie eingegangen wird und verschiedene Regulierungsinitia-

23 Lösch et al. 2008, S. 35.

24 EurActiv 2007 a.

25 Lösch et al. 2008, S. 18. 
tiven anlaufen, kommen gleichzeitig stetig neue Nanotechnologie-Produkte auf den Markt. Ab dem Jahr 2014 sollen Produkte, die Nanoteilchen oder -materialien enthalten, nach einem im Juli 2011 getroffenen Beschluss des EU-Parlaments schließlich auch gekennzeichnet sein. Wie die Kennzeichnung aussehen soll und welche Materialien unter die Kennzeichnungspflicht fallen, ist allerdings noch offen. Die verschiedenen Strategien der Äquivalenzierung von Artikulationen sind daher insofern erfolgreich, als die Nanotechnologie-Entwicklung bisher nicht durch strenge gesetzliche Regulierungen eingeschränkt wird. Gleichzeitig wirken diese Strategien integrierend, da die Bedenken gehört werden und viele Akteure an der Entwicklung von freiwilligen oder gesetzlichen Risikoregulierungen beteiligt sind. Wenn es allerdings auch mittelfristig kaum zu robusteren Risikoregulierungen kommen sollte, könnten diese Strategien stärkeren Gegenwind erhalten (siehe unten).

\section{Legitime Differenz und Nano-Dialoge}

Andere Strategien innerhalb des Nanotechnologie-Diskurses, die hier unter dem Strategie-Cluster der legitimen Differenz gefasst werden sollen, zielen darauf ab, antagonistischen Forderungen oder Subjektpositionen ihren für die Akzeptanz der Nanotechnologie bedrohlichen Charakter zu nehmen. Politische Forderungen mit einer der Nanotechnologie gegenüber (potenziell) antagonistischen Position werden hierdurch zu einfachen Widersprüchen abgemildert und als legitime Differenzen deklariert. Wenn »alte Feinde ... zur Überprüfung der Risiken von Nanotechnologien zusammen[arbeiten] «, ${ }^{26}$ wird eine erfolgreiche Strategie der legitimen Differenz ersichtlich.

Im Nanotechnologie-Diskurs werden Strategien der legitimen Differenz angewendet, um kritische Akteure zu integrieren und insbesondere die Öffentlichkeit von der Nanotechnologie zu überzeugen. Wie zu Anfang ausgeführt wurde, ist das Wissen um die Nanotechnologie innerhalb der Gesellschaft recht begrenzt. Die meisten Menschen können mit dem Begriff Nanotechnologie wenig anfangen, und es gibt weder ausgeprägte positive noch negative Ressentiments gegenüber der Nanotechnologie - insgesamt aber eine eher offene Grundhaltung. Auch wenn der Gentechnik-Diskurs nicht ohne weiteres mit dem Nanotechnologie-Diskurs verglichen werden kann, war doch die Ausgangssituation eine ähnliche. Allerdings gab es zu Beginn des Gentechnik-Diskurses keine Diskussion in der Öffentlichkeit um mögliche Risiken. Vor allem die Industrie hielt problematische Studien zurück, und kritische Positionen fanden kaum Beachtung. Stattdessen wurde davon ausgegangen, dass die KonsumentInnen die Gentechnik-Produkte umstandslos annehmen würden. In den 1990er Jahren machten die Gentechnik-Protagonisten dann die Erfahrung, dass eine neutrale bis tendenziell positive Grundhaltung einer Bevölkerung, die relativ wenig über die Gentechnik Bescheid wusste, schlagartig in starke Ablehnung umschlagen kann. Für Europa konnte konstatiert werden, dass die »Kriege um das Ansehen [der

26 EurActiv $2007 \mathrm{~b}$.

Leviathan, 40. Jg., 1/2012 
Gentechnik] von der Industrie einer nach dem anderen verloren wurden « 27 und gentechnisch veränderte Lebensmittel im Wert fielen, anstatt Wert zu produzieren.

Im Nanotechnologie-Diskurs finden sich regelmäßig Verweise auf diese für die Gentechnik-Industrie recht schmerzliche Erfahrung. Auch das BMU konstatiert: »Bei Technologien wie der Biotechnologie sind in Deutschland in der Vergangenheit Fehler gemacht worden, die bis heute zu erheblichen Akzeptanzdefiziten gegenüber den Technologien führen ${ }^{2}{ }^{28}$

Aus dieser Erfahrung wurde insofern gelernt, als erkannt wurde, dass eine uninformierte Öffentlichkeit ein Risiko für die Nanotechnologie-Entwicklung darstellt. Hinsichtlich der Gentechnik hatte man zwar festgestellt, dass die Risikodiskussion eher zur Akzeptanzsteigerung beitrug, doch diese Erkenntnis setzte sich erst zu einem Zeitpunkt durch, als die Stimmung in vielen Ländern bereits gekippt war. Für den Nanotechnologie-Diskurs wurde daraus geschlussfolgert, »dass die Wissenschaftsgemeinschaft ihre Kommunikationsfähigkeit verbessern muss «. ${ }^{29}$

Das Vertrauen der Bevölkerung in die Sicherheit der Nanotechnologie und die öffentliche Akzeptanz werden also als Voraussetzungen für die NanotechnologieEntwicklung und -kommerzialisierung gesehen. Auch das Bundesamt für Strahlenschutz bezieht sich auf den Konflikt um die Gentechnik, wenn es vor den negativen Konsequenzen einer fehlenden öffentlichen Diskussion warnt: »Das Versäumnis, die Öffentlichkeit an der strategischen Entwicklung neuer Technologien zu beteiligen und die Sorgen der Öffentlichkeit zu berücksichtigen, ... führt zu Misstrauen gegenüber öffentlichen Institutionen $"{ }^{30}$ Kurz: Für die Protagonisten der Nanotechnologie geht es darum, eine solche Entwicklung zu verhindern.

In vielen Regierungsprogrammen wurde die Nanotechnologie-Forschung daher von Programmen zur Evaluation der gesellschaftlichen Dimension der Nanotechnologie-Entwicklung begleitet. Weiterhin wurde eine Vielzahl von Dialogen mit und innerhalb der Bevölkerung initiiert, um die BürgerInnen zu einer positiven Einstellung gegenüber der Nanotechnologie zu motivieren. Während diese Dialoge für die einen das Ziel haben, »Missverständnisse «, »Blockaden « und "grundlose « Ängste in der Bevölkerung gegenüber der Nanotechnologie zu mindern, kann die Dialogstrategie als solche nicht auf eine solche Position reduziert werden. Die verstärkten Kommunikationsbemühungen sind vielmehr Teil einer umfassenderen Strategie. Auf die EU bezogen geht diese zurück auf die Lissabon-Agenda, in der die Schaffung einer »wissensbasierten " Gesellschaft gefordert wird. Teil dieses Konzepts ist die Vorstellung, dass die EU-BürgerInnen die gesellschaftlichen Entwicklungen - innerhalb eines bestimmten Rahmens - aktiv mitgestalten sollen. Hier wird Technologieentwicklung durchaus als demokratischer Prozess verstanden. Es geht also nicht nur um reine Akzeptanzbemühungen, vielmehr wird der Prozess selbst als wertvoll gesehen, sowohl in Hinsicht auf die Stärkung einer demokratischen (west-

27 Mitsch, Mitchell 1999, S. 3.

28 BMU 2005, S. 5.

29 Europäische Kommission 2004, S. 23.

30 Bundesamt für Sicherheit in der Informationstechnik 2007, S. 168. 
lich-europäischen) Kultur als auch im Hinblick auf den Ausbau einer (westlicheuropäischen) Wertegemeinschaft.

Allerdings ist dieser Prozess insofern selektiv, als dass die Notwendigkeit von Technologieentwicklung nicht in Frage gestellt wird. Letztlich geht es um »die Erhöhung der gesellschaftlichen Akzeptanz von Wissenschaft und Forschung « ${ }^{31}$ im Allgemeinen und im Speziellen um eine gefestigte Basis für die gesellschaftliche Akzeptanz der Nanotechnologie. ${ }^{32}$ Die Frage über Sinn und Zweck von technologischem Fortschritt bzw. nanotechnologischer Forschung wird nicht gestellt und steht auch nicht zur Diskussion.

Auf der Grundlage dieser neuen Artikulationsstrategien liefen in den letzten Jahren verschiedene Initiativen an. Im Jahr 2005 wurde ein zweijähriger, von der EUKommission finanzierter und unter der Federführung des Wuppertal Instituts durchgeführter Nanologue begonnen. Weiterhin wurde unter dem Sechsten Forschungsrahmenprogramm der Europäischen Union das Projekt Nanodialogue Enhancing dialogue on Nanotechnologies and Nanosciences in society at the European level gestartet. Es war das erste groß angelegte europäische Kommunikationsprojekt seiner Art, durchgeführt vor allem zum Zweck der Vertrauensbildung und Akzeptanzschaffung. Auch in der Bundesrepublik gab es verschiedene Kommunikationsinitiativen. Mit dem Ziel, möglichst viele Menschen zu erreichen, ist in Deutschland seit dem Jahr 2004 zum Beispiel der nanoTruck, ein mobiles Kommunikations- und Ausstellungszentrum, unterwegs.

Ende 2005 wurde vom Bundesumweltministerium (BMU), dem Umweltbundesamt (UBA) und der Bundesanstalt für Arbeitsschutz und Arbeitsmedizin (BAuA) ein Dialog zur Bewertung von synthetischen Nanopartikeln in Arbeits- und Umweltbereichen in Bonn organisiert, an dem etwa 170 Fachleute aus Unternehmen, Wissenschaft, Verwaltung, Umwelt-, Verbraucher- und Sozialverbänden sowie VertreterInnen der EU-Kommission und der OECD teilnahmen. Auch hier ging es darum, »Fehler zu vermeiden, die in der Vergangenheit bei anderen Entwicklungen - wie etwa der Gentechnik - gemacht wurden ",33 und frühzeitig über die Chancen und Risiken der Nanotechnologie zu diskutieren.

Resultat dieses Dialogs war die Einrichtung einer Steuerungsgruppe, die im Laufe des Jahres 2006 als sogenannte Nanokommission gebildet wurde. Sie setzte sich zusammen aus VertreterInnen von Unternehmen, Umwelt- und Verbraucherschutzverbänden, Arbeitnehmerschaft und staatlichen Stellen. Ende 2006 wurde dann vom Bundesumweltministerium in Zusammenarbeit mit der Nanokommission ein über zwei Jahre laufender NanoDialog gestartet, dessen Ziel sein sollte, »eine nachhaltige und zukunftsfähige Entwicklung und Nutzung der Nanotechnologie ... zu unterstützen $"{ }^{34}$ In dem Dialog, in dem es um die Chancen und Risiken von Nano-

31 Europäische Kommission 2007, S. 84.

32 Bonazzi 2007, S. 10.

33 BMU et al. 2005.

34 Nanokommission 2007.

Leviathan, 40. Jg., 1/2012 
materialien ging, sollten Handlungsempfehlungen für die Behörden erarbeitet werden.

Vom Bundesinstitut für Risikobewertung (BfR) wurde im Jahr 2006 gemeinsam mit dem Unabhängigen Institut für Umweltfragen (UfU) sowie dem Institut für ökologische Wirtschaftsforschung eine sogenannte Verbraucherkonferenz durchgeführt. Hier ging es um die Erprobung neuer Wege in der Risikokommunikation. ${ }^{35}$ So war es das erste Mal, dass eine Behörde in Deutschland diese Art der Risikokommunikation wählte. Ähnliche Verbraucherkonferenzen gab es auch in anderen Ländern, beispielsweise in Frankreich, der Schweiz, den Niederlanden, Dänemark, Finnland und den USA.

Auf eine ganz ähnliche Weise erprobt die Privatwirtschaft neue Wege der Risikokommunikation. So organisierte das Forum econsense für Nachhaltige Entwicklung der Deutschen Wirtschaft gemeinsam mit BASF und Siemens in den Jahren 2005 und 2006 Dialogveranstaltungen unter dem Motto »Chancen nutzen - Risiken managen. Weichen stellen für eine nachhaltige Nanotechnologie«. Von 2004 bis 2006 lief der Risiko-Dialog Comparative Challenge of Nanomaterials - CONANO an. Dieser Dialog fand zwischen Novartis International AG, Ciba Specialty Chemicals Inc., dem Freiburger Öko-Institut und dem Wiener Ökologie-Institut statt; Aufgabe war es, eine gemeinsame Nutzen-Risiko-Analyse zu entwickeln.

Interessanterweise waren nach vorliegenden Berichten die Ergebnisse der meisten der Verbraucherkonferenzen eher kritisch in Bezug auf die derzeit geltenden Regulierungen der Nanotechnologie-Entwicklung und betrachteten sie als zu lasch und vage. Aus den verschiedenen Konferenzen kamen ähnliche Vorschläge zur Verbesserung der derzeitigen Regulierungen. Trotz dieser Kritik an den bestehenden Regulierungen wurden die verschiedenen Dialogstrategien als Erfolg gesehen: » Man ist im Gespräch «. ${ }^{36}$ Die Öffentlichkeit ist zwar immer noch unzureichend über die Nanotechnologie informiert. Doch die meisten kritischen zivilgesellschaftlichen Organisationen sind auf die eine oder andere Weise am Kommunikationsprozess beteiligt: »Die grüne Gentechnik war auf einmal da und man musste sich überlegen, wie reagieren und wir haben erst einmal gesagt: Stopp! Halt! (...) Bei der Nanotechnologie sind wir, zumindest von BUND Seite aus, in die Diskussionen shineingerutscht $\longleftarrow$. Wir sind also in einem Dialog ... Das bewirkt, dass sich die Bevölkerung, auch wenn es nur ein eingeschränkter Fachkreis ist, anders mit der Thematik beschäftigt «. ${ }^{37}$ Durch die Integration von Akteuren, die im Gentechnik-Diskurs Teil des antagonistischen Lagers waren, wurde eine solche Lagerbildung in Bezug auf die Nanotechnologie bislang verhindert: »Bei der Gentechnik waren eher Pro-Contra-Positionen festgelegt: Es gab die guten Umweltschützer und die böse Industrie. Bei der Nanotechnologie ist es nicht so einfach «. .38

35 Siehe BfR 2006.

36 Natalie Eckert am 11. Juli 2008 in einem Interview mit dem BUND.

37 Ebd.

38 Ebd. 
Die meisten zivilgesellschaftlichen Akteure scheint es bislang kaum zu stören, dass bei den Dialogen die Nanotechnologie selbst gar nicht in Frage gestellt wird, sondern nur thematisiert wurde, wie die Nanotechnologie-Entwicklung ausgestaltet werden soll und dass am »Wie "zwar alle mitreden dürfen, Gestaltungsrechte aber nicht gewährt werden. Einige der Verbraucherkonferenzen äußerten sich zwar enttäuscht, dass ihre Empfehlungen an die Politik kaum zur Kenntnis genommen wurden. Insgesamt werden die Dialoge jedoch eher positiv bewertet, und es gibt nur wenige Stimmen, die die Dialog-Strategie insgesamt kritisieren.

\section{Grüne Nanotechnologie}

Eine weitere, äußerst erfolgreiche Strategie besteht darin, eine Gemeinsamkeit - eine Äquivalenz - zwischen Nanotechnologie und Nachhaltigkeit zu artikulieren. Die Forschungen etwa zu umweltfreundlichen Antriebstechnologien, zu Umwelttechnologien zur Gewinnung von sauberem Trinkwasser, zur Steigerung der Energieeffizienz von Solaranlagen und zu Energiespeichertechnologien wecken bei vielen Akteuren Hoffnungen auf große Umweltentlastungspotenziale. Die Nanotechnologie verspricht eine der Säulen der Green Economy zu werden. ${ }^{39}$ Angesichts dieser Nachhaltigkeitspotenziale beziehen sich die meisten der Umweltorganisationen und grünen Parteien, von denen praktisch alle eine ablehnende Haltung gegenüber der Gentechnik einnehmen, positiv auf die Nanotechnologie. So betont die NGO Friends of the Earth USA im Jahre 2007: »Friends of the Earth is not anti-nano! There will be some good applications of nanotechnology « ${ }^{40}$ Kritische Stimmen gegenüber den Hoffnungen auf das Nachhaltigkeitspotenzial sind dagegen eher rar: "... es ist theoretisch durchaus möglich, dass diese Technik auch ökologischen Nutzen haben kann (...). Gleichzeitig wissen wir aus der Geschichte, dass neue Technologien, die in eine nicht wirklich gerechte soziale Ordnung hineingebracht wurden, jene mächtiger und reicher gemacht hat, die Macht und Reichtum hatten « ${ }^{41}$ Auf allgemeiner Ebene setzt die Kritik des BUND an: »Die vordergründige Fokussierung auf technische Lösungen, die als die Zukunftstechnologien verkauft werden, verstellt ... den Blick auf zukunftsfähige Entwicklungen, deren Lösungen an den wirklichen Ursachen - wie den nicht zukunftsfähigen Lebensstilen der hoch entwickelten Industrienationen - ansetzen müssen «. ${ }^{42}$ Die meisten Akteure im Nanotechnologie-Diskurs äußern jedoch große Erwartungen in das behauptete Nachhaltigkeitspotenzial der Nanotechnologie.

\section{Gegen-hegemoniale Strategien}

Diskurstheoretisch gesehen zielen gegen-hegemoniale Strategien auf eine antagonistische Zweiteilung des Diskursraums mit der Absicht der Destabilisierung des

39 Vgl. ETC 2010, S. vi.

40 Interview mit Erich Pica am 12. März 2007 in Washington, D.C.

41 Mooney 2006, S. 39.

42 BUND 2007, S. 10.

Leviathan, 40. Jg., 1/2012 
hegemonial strukturierten Diskurses. Als erster kritischer Akteur unternahm die ETC Group - eine international agierende NGO - gemeinsam mit dem International Center for Technology Assessment (ICTA) seit 2002 verschiedene Versuche, den hegemonialen Pro-Nanotechnologie-Diskurs zu destabilisieren. Die ETC Group stellt auch weiterhin das »Flaggschiff « der nanotechnologiekritischen zivilgesellschaftlichen Organisationen (Civil Society Organisation, CSO) dar, auch wenn sie ihre strategische Ausrichtung seit 2007 auf einen bestimmten Bereich der Nanotechnologie - die synthetische Biologie - fokussiert hat. Die meisten anderen kritischen CSOs folgten erst im Jahr 2005/2006, und viele sind weiterhin auf Positionssuche.

Im Jahr 2002 riefen die ETC Group, Greenpeace International und andere international agierende CSOs gemeinsam zu einem Moratorium gegenüber der Freisetzung von hergestellten Nanomaterialien auf. Mitte 2006 reichten acht Verbraucherorganisationen eine Petition bei der US-Regierung gegen die Einführung und für den Rückruf von Kosmetikprodukten, die synthetische Nanopartikel enthalten, ein. Mitte 2007 veröffentlichte eine Koalition, bestehend aus 44 verschiedenen Verbraucher-, Umwelt-, Gewerkschaftsorganisationen und anderen CSOs aus sechs Kontinenten, ein Statement of Principles for the Oversight of Nanotechnologies and Nanomaterials. ${ }^{43}$ In dieser Stellungnahme wird ein starkes, umfassendes Risikomanagement eingefordert. Ende 2010 wiederholten und bekräftigten 46 Nichtregierungsorganisationen aus Europa, den USA, Südamerika und Asien ihre Forderungen.

Die kritischen CSOs haben mit verschiedenen Spezifika der Nanotechnologie zu kämpfen: Erstens stellt die artikulierte Universalität der Nanotechnologie ein Problem dar: »Für die Anti-Gentechnik-Bewegung ist es relativ einfach, ihre Ablehnung zu formulieren, da die Gentechnik sehr spezifische Techniken und Risiken beinhaltet. Bei der Nanotechnologie handelt es sich jedoch um sehr verschiedene Techniken, deren einzige Gemeinsamkeit ist, dass sie auf dem gleichen Maßstab agieren. Folglich stehen NGOs vor dem Problem, die Risiken dieser sehr unterschiedlichen Techniken zu formulieren « ${ }^{44}$

Da die Nanotechnologie für ein Amalgam von verschiedenen technologischen Entwicklungen steht, stellt sich die Frage, gegen was gekämpft werden soll. Manche CSOs reagieren auf diese Herausforderung, indem sie sich eines begrenzten Bereichs annehmen. So fokussiert Friends of the Earth in ihrer Kampagne auf Nanomaterialien, die in Kosmetika verwendet werden. Der BUND beschäftigt sich vor allem mit der Problematik der Nanotechnologie im Lebensmittelsektor. Andere Organisationen und vor allem die ETC Group versuchen, die gesamtgesellschaftliche Dimension der Nanotechnologie in den Blick zu nehmen. Die ETC Group ist bislang die einzige Organisation, die den »Kampf um die Erzählung « 45 umfassend in Angriff genommen hat: In ihren ersten Publikationen zu diesem Thema wird die Nanotech-

43 ICTA et al. 2007.

44 E-Mail Interview mit Jim Thomas von der ETC Group am 21. März 2007.

45 Interview mit Patrick Reinsborough von SmartMeme am 7. März 2007 in San Francisco. 
nologie als "Atomtechnologie « bezeichnet. Ihr wahrscheinlich einflussreichster Report lautet: »The Big Down: Atomtech - Technologies Converging at the Nanoscale «. ${ }^{46}$ Nicht nur wird in diesen Publikationen ein Vergleich mit der Gentechnik gezogen, also im Laclau'schen Sinne eine Äquivalenz zur Gentechnik hergestellt es wird zum Beispiel vom »atomically modified rice $" 47$ gesprochen - und betont, dass die Nanotechnologie die Möglichkeiten der Gentechnik um ein Vielfaches potenziert. Auch sollten mit dem Begriff Atomtechnologie Assoziationen mit dem negativ besetzten Begriff der Atomenergie geweckt werden.

Der Versuch der Begriffsumwidmung misslang jedoch, und bereits in den Publikationen des folgenden Jahres wurde der Begriff Atomtechnologie wieder fallengelassen. Dafür wird sich anderer Begriffe wie beispielsweise der Tsunami-Metapher bedient: »Die Nanotechnologie-Entwicklung nimmt rasant an Bedeutung zu, während sie zugleich unter der Oberfläche [der gesellschaftlichen Wahrnehmung] verbleibt und vor ihrem Aufprall praktisch unbemerkt voranschreitet. Wenn die Nanotechnologie-Welle die Küste erreicht, wird es rasante, gewaltige und verheerende Veränderungen zur Folge haben «. ${ }^{48}$ Außer der ETC Group und einigen Gruppen aus dem Kontext sozialer Bewegungen in Frankreich gibt es allerdings derzeit so gut wie keinen Akteur, der gegen-hegemoniale Strategien verfolgte.

\section{Erfolgreiche Nano-Governance?!}

Innerhalb des Nanotechnologie-Diskurses ist es bislang noch nicht zur Bildung eines antagonistischen Lagers von Akteuren gekommen. An einigen Stellen regt sich allerdings Unmut gegenüber der derzeitigen Governance der Nanotechnologie. Noch gibt es zwar verhältnismäßig wenige kritische Akteure, doch die Kritik kommt von sehr verschiedenen Seiten und Akteuren. Bezogen auf die Entwicklung eines freiwilligen Verhaltenskodexes, kritisiert beispielsweise die Evangelische Kirche in Deutschland (EKD), dass angesichts der vielen offenen Fragen zu möglichen Risiken der Nanotechnologie ein freiwilliger Regulierungsrahmen unzureichend sei. ${ }^{49}$ Vielmehr wird ein verbindlicher Regulierungsrahmen angemahnt, der auch auf mögliche Veränderungen von Menschenbildern Bezug nehmen soll. Daneben wird die Begrenzung des Verhaltenskodexes auf die Forschung problematisiert und eine Ausweitung der Regulierung auf die gesamte Entwicklung und Produktion gefordert.

Auch die Rückversicherer melden sich mit einer Mahnung zur strengeren Regulierung zu Wort: »Die Assekuranz ist besorgt. Nicht, weil sich im Zuge neuer technologischer Entwicklungen erfahrungsgemäß neue Schadenszenarien auftun, sondern weil das Ausmaß dieser potenziellen Schäden falsch oder gar nicht eingeschätzt werden kann «. 50

46 ETC 2003.

47 ETC 2004.

48 ETC 2005, S. vii.

49 EKD 2007.

50 Swiss Re 2004, S. 39.

Leviathan, 40. Jg., 1/2012 
Die Gewerkschaften nehmen klassischerweise eine ambivalente Position ein: »Das Entwicklungspotenzial der Nanotechnologie ist riesig. Einen nachhaltigen Beitrag zur Sicherung und Schaffung von Arbeitsplätzen am Produktionsstandort Deutschland kann sie aber nur dann leisten, wenn einer konsequenten Risikoerkennung und -vermeidung absolute Priorität eingeräumt werden «. ${ }^{51}$ Auch VerbraucherschutzOrganisationen treten verstärkt im Nanotechnologie-Diskurs in Erscheinung. Hier werden vor allem umfassende Informationen für die Verbraucher hinsichtlich möglicher Risiken, eine Kennzeichnungspflicht und eine staatliche Zulassung und Registrierung von nanotechnologischen Anwendungen sowie Nanomaterialien und -produkten angemahnt.

Anfang 2008 beschloss die britische Soil Association, die für die Zertifizierung von ca. 56 Prozent der ökologisch arbeitenden landwirtschaftlichen Betriebe und ca. 70 Prozent der in Großbritannien verkauften biologisch angebauten Lebensmittel zuständig ist, nanotechnologische Produkte nicht als »Bio « zu zertifizieren und zusätzlich zu dem Standard "gentechnikfrei « nun einen Standard »nanotechnikfrei « einzuführen. Interessant ist der Beschluss der Soil Association auch aus dem Grund, weil diese bereits in die Nanotechnologie-Dialoge integriert zu sein schien. Sie war Teil einer Arbeitsgruppe zur Entwicklung eines freiwilligen Nanotechnologie-Produktlabels. Nachdem die Informationspolitik der Industrie jedoch als unzureichend und der Prozess insgesamt als zu langsam empfunden wurde, sah sich die Soil Association gezwungen, im Alleingang zu handeln. In Stellungnahmen der Soil Association wird ein direkter Vergleich mit der Gentechnik hergestellt: »Wie bei der Gentechnik gibt es ein Rennen um Patente ... Wie bei der Gentechnik sind die Risiken der Nanotechnologie unbekannt, ungeprüft und nicht vorhersehbar. (...) Wie bei der Gentechnik sind manche Risiken so gewaltig, dass sie nicht versichert werden können « ${ }^{52} \mathrm{Im}$ Jahr 2010 reagierten einige Naturkosmetikfirmen, indem sie auf den Einsatz von Nanopartikeln verzichteten und ihre Produkte mit dem Label »Nanofrei « kennzeichneten. Im August 2011 beschloss der Öko-Verband Naturland vorerst -, auf die Verwendung von Nanomaterialien in Lebensmitteln, Kosmetika und auch in den Verpackungen zu verzichten.

\section{Ausblick}

Mit Rückgriff auf die Hegemonietheorie von Laclau und Mouffe wurde in diesem Beitrag die strategische Dimension im Diskurs um die Nanotechnologie analysiert. Es wurde aufgezeigt, dass Protagonisten der Nanotechnologie aus dem GentechnikDiskurs gelernt haben. Viele Akteure haben erkannt, dass die Nanotechnologie nur wirklich erfolgreich sein wird, wenn sie einen positiv besetzten Platz im Alltagsverstand (Gramsci) der Menschen erhält. Auch wenn sich die Strategien unterscheiden, also keine einheitliche Linie existiert, liegt doch ein eindeutiger Schwerpunkt in dem Versuch, den Konsens mit den wichtigsten gesellschaftlichen Akteuren zu erhalten.

51 IG Metall 2007, S. 2.

52 Soil Association 2005, S. 1. 
Diese Strategien haben bisher dazu geführt, dass kaum Akteure ausgemacht werden können, die der Nanotechnologie gegenüber eine grundsätzlich ablehnende Haltung einnehmen - eine »Anti-Nanotechnologie-Bewegung « ist derzeit nicht in Sicht. Kritische Stimmen beanstanden zwar das Wie, aber nicht das Ob der NanotechnologieEntwicklung.

Analytisch wurde zwischen zwei Dimensionen innerhalb einer Hegemonie unterschieden, die Gramsci Transformismus und expansive Hegemonie genannt hat. Transformismus beschreibt eine Form der Hegemonie, in der in der Tendenz relevante gesellschaftliche Kräfte in das hegemoniale Projekt integriert werden konnten und es dadurch möglich ist, dieses auch ohne Zustimmung weiter Teile der Bevölkerung durchzusetzen. In der Bevölkerung herrscht dann ein weitgehend passiver Konsens vor. Bei der expansiven Hegemonie hingegen gelingt es, das hegemoniale Projekt so zu kontextualisieren, dass dieses tendenziell von der breiten Masse der Bevölkerung als wünschenswert angesehen wird und ein aktiver Konsens vorliegt. Eine solche Hegemonie basiert auf aktiver Zustimmung, ist daher umfassend und expansiv hegemonial. Beide Formen der Hegemonie sind nicht als fixierte Zustände zu verstehen, sondern stellen Prozesse dar, die Veränderungen unterliegen.

Die gesellschaftliche Stimmung kann derzeit in Richtung Transformismus gedeutet werden. Wichtige gesellschaftliche Akteure sind in das Nanotechnologie-Projekt integriert, und auch viele vormals im Gentechnik-Diskurs antagonistische Kräfte konnten gewonnen werden. Gleichzeitig wird dieser Konsens (noch) nicht von der breiten Bevölkerung getragen. Eine Situation des Transformismus ist instabiler als eine expansive Hegemonie. Unter bestimmten Voraussetzungen kann diese aber Bestand haben, etwa wenn das Thema von der breiten Bevölkerung nicht wahrgenommen wird oder wenn es nicht als bedeutungsvoll erkannt wird, wenn also eine gewisse Gleichgültigkeit vorherrscht.

Aus Sicht der Befürworter der Nanotechnologie kann bislang also durchaus von einer erfolgreichen Governance gesprochen werden. Auch aus demokratietheoretischer Sicht scheint es sich auf den ersten Blick um einen gelungenen Prozess zu handeln: Alle wichtigen Akteure sitzen gemeinsam an einem Tisch und sind an den Dialogen beteiligt. Bei genauerem Hinsehen stellt sich die Situation allerdings anders dar. Zum einen schreitet die Nanotechnologie-Entwicklung voran, ohne dass das Gefahrenpotenzial bestimmter involvierter Technologien für Mensch und Umwelt abgeschätzt werden kann. Das Vorsorgeprinzip wird missachtet, und gesundheitliche und soziale Fragen werden ausgeblendet.

Angesichts dessen scheint eine Politisierung des Diskurses um die Nanotechnologie durch eine stärkere Einbeziehung der Konfliktdimension wichtig und notwendig: »Es wird heute viel von `Dialog und `Deliberation ‘ geredet, aber was bedeuten diese Wörter auf dem Gebiet des Politischen, wenn keine echte Wahlmöglichkeit besteht und die Diskussionsteilnehmer sich nicht zwischen klar voneinander abgehobenen Alternativen entscheiden dürfen? "53 Es waren die starken Konflikte und gesellschaftlichen Auseinandersetzungen um die Gentechnik, die dazu führten, dass breite Teile der Bevölkerung an den Diskussionen teilnahmen. Insbesondere die konträr

53 Mouffe 2007, S. 10.

Leviathan, 40. Jg., 1/2012 
und antagonistisch zueinander stehenden Positionen haben den Diskurs um die Gentechnik belebt. Hauptsächlich aus diesem Grund ist es in vielen Ländern zu einem eher vorsichtigen Umgang mit der Gentechnik gekommen.

Der Diskurs um die Nanotechnologie ist bislang vor allem durch eine "postpolitische" Governance charakterisiert: ${ }^{54} \mathrm{Da}$ vermeintlich alle Akteure an einem Strang ziehen - für eine "grüne Nanotechnologie «, für effizientere Technologien, für die Stärkung der Wettbewerbsfähigkeit, für eine Green Economy -, wird die Konfliktdimension derzeit bewusst und strategisch ausgeblendet. Auch die sozioökonomische Dimension der Nanotechnologie-Entwicklung wird nicht thematisiert - zu offensichtlich würde sich zeigen, dass die technologischen Entwicklungen, die unter dem Begriff Nanotechnologie gefasst werden, nicht nur potenzielle gesundheitliche Gefahren beinhalten, sondern auch verheerende Auswirkungen auf die weniger industrialisierten Länder haben könnten, wenn etwa natürliche Rohstoffe wie Kautschuk, Baumwolle und Jutefasern durch synthetisch hergestellte Rohstoffe ersetzt werden würden. ${ }^{55}$ Eine Politisierung des Diskurses wäre allerdings notwendig, damit die rasanten technischen und technologischen Entwicklungen, die unter dem Begriff Nanotechnologie gefasst werden, verlangsamt und demokratisiert werden können. Durch eine »Entschleunigung « der Entwicklung könnte Zeit gewonnen werden, um die Auswirkungen der Nanotechnologie auf die Gesellschaft zu diskutieren. Das hieße auch, kritisch zu hinterfragen, welche sozioökonomischen Veränderungen zu erwarten sind und welche technologischen Entwicklungen überhaupt gesellschaftlich gewünscht werden.

\section{Literatur}

BfR 2006. Verbraucherkonferenz Nanotechnologie. Hintergrundinformation für Journalisten. http://www.bfr.bund.de/cd/8551 (Zugriff vom 15.12.2011).

BMU 2005. Synthetische Nanopartikel - Entwicklungschancen im Dialog. http://www.bmu.de/files/pdfs/allgemein/application/pdf/themenpapier_nanopartikel.pdf (Zugriff vom 09.01.2012).

BMU; BAuA; UBA 2005. Dialog über Chancen und Risiken synthetischer Nanopartikel beginnt. Presseinformation. http://www.bmu.de/pressearchiv/15_legislaturperiode/pm/36132.php (Zugriff vom 15.12.2011).

Bonazzi, Matteo 2007. Strategy for communication outreach in nanotechnology. Working paper Resulting from the Workshop on Strategy for Communication Outreach in Nanotechnology. Brussels: European Commission. ftp://ftp.cordis.europa.eu/pub/nanotechnology/docs/nano _outreach_final.pdf (Zugriff vom 15.12.2011).

Bullis, Kevin 2006. »Angst vor Nano im Regal «, in Technology Review vom 12.04.06. http://w ww.heise.de/tr/artikel/71931 (Zugriff vom 15.12.2011).

BUND 2007. BUND-Position "Für einen verantwortungsvollen Umgang mit der Nanotechnologie«. Eine erste Diskussionsgrundlage am Beispiel der Nanopartikel. http://www.bund.n et/lab/reddot2/pdf/bundposition_nano_03_07.pdf (Zugriff vom 15.12.2011).

BUND 2009. EU-Kosmetikverordnung nachbessern. http://www.bund.net/fileadmin/bundnet/p dfs/nanotechnologie/20090129_nanotechnologie_kosmetikverordnung_forderungen.pdf (Zugriff vom 15.12.2011).

54 Mouffe 2007.

55 Vgl. ETC 2005; Wullweber 2006. 
Bundesamt für Sicherheit in der Informationstechnik 2007. Nanotechnologie. https://www.bsi. bund.de/SharedDocs/Downloads/DE/BSI/Publikationen/Studien/Nanotechnologie/Nanotec hnologie_pdf.pdf?_blob=publicationFile (Zugriff vom 09.01.2012).

Burson-Marsteller AG 2004. Nanotechnology in need of successful communication. http://ww w.b-m.ch/fileadmin/Burson-Marsteller/Publikationen/B-M_Information_/B-M_Information _Nanotechnology.pdf (Zugriff vom 15.12.2011).

Canzler, Weert; Marz, Lutz 2011. "Wert und Verwertung neuer Technologien. Das Beispiel der Wasserstoff- und Brennstoffzellentechnologien ", in Leviathan 39, 2, S. 223-246.

DEFRA (Department for Environment Food and Rural Affairs) 2006. UK voluntary reporting scheme for engineered nanoscale materials. http://archive.defra.gov.uk/environment/qualit $\mathrm{y} /$ nanotech/documents/vrs-nanoscale.pdf (Zugriff vom 15.12.2011).

EKD 2007. Verhaltenskodex zum verantwortungsvollen Umgang mit der Nanotechnologie. Stellungnahme zur Entwicklung eines Verhaltenskodexes vom 27.9.2007. http://www.ekd. de/bevollmaechtigter/themen/070927_nanotechnologie.html (Zugriff vom 15.12.2011).

ETC 2003. The big down: atomtech - technologies converging at the nanoscale. http://www.et cgroup.org/upload/publication/171/01/thebigdown.pdf (Zugriff vom 15.12.2011).

ETC 2004. Jazzing up jasmine: atomically modified rice in Asia? News Release. http://www.etc group.org/en/materials/publications.html?pub_id=117 (Zugriff vom 15.12.2011).

ETC 2005. The potential impact of nano-scale technologies on commodity markets: the implications for commodity dependent developing countries. http://www.etcgroup.org/upload/p ublication/45/01/southcentre.commodities.pdf (Zugriff vom 09.01.2012).

ETC 2010. The big downturn? Nanogeopolitics. http://www.etcgroup.org/en/node/5245 (Zugriff vom 15.12.2011).

EurActiv 2007 a. EU führt Verhaltenskodex für Nanotechnologie ein. http://www.euractiv.com/ de/wissenschaft/eu-fhrt-verhaltenskodex-nanotechnologie/article-165736 (Zugriff vom 15.12.2011).

EurActiv 2007 b. Alte Feinde arbeiten zur Überprüfung der Risiken von Nanotechnologien zusammen. http://www.euractiv.com/de/wissenschaft/alte-feinde-arbeiten-berprfung-risiken-n anotechnologien-zusammen/article-165050 (Zugriff vom 15.12.2011).

Europäische Kommission 2004. Auf dem Weg zu einer europäischen Strategie für Nanotechnologie. Luxembourg: Office for Official Publications of the European Communities.

Europäische Kommission 2007. Europäischer Forschungsraum: Neue Perspektiven - Grünbuch. Luxembourg: Office for Official Publications of the European Communities.

Europäische Kommission 2008. Europäische Kommission verabschiedet Verhaltenskodex für verantwortungsvolle Forschung in Nanowissenschaft und -technologie. http://europa.eu/ra $\mathrm{pid} /$ pressReleasesAction.do? reference=IP/08/193\&format=PDF\&aged=1\&language=DE\& guiLanguage $=$ en (Zugriff vom 15.12.2011).

European Commission 2004. Nanotechnologies: a preliminary risk analysis on the basis of a workshop organized in Brussels on 1-2 March 2004 by the Health and Consumer Protection Directorate General of the European Commission. Luxembourg: Office for Official Publications of the European Communities.

European Commission 2007. Towards a code of conduct for responsible nanosciences and nanotechnology research. http://ec.europa.eu/research/consultations/pdf/nano-consultation_e n.pdf (Zugriff vom 15.12.2011).

Feder, Barnaby J. 2006. "Nanotech food is ten times scarier than genetically engineered «, in New York Times online vom 10.12.2006. http://www.blackherbals.com/nanotech_food_is_ten_ times_scarier_than_GE.htm (Zugriff vom 15.12.2011).

Gramsci, Antonio. 1971. Selections from the Prison Notebooks. London: Lawrance \& Wishart. Gramsci, Antonio 1991. Gefängnishefte. Hamburg u.a.: Argument Verlag.

Hajer, Maarten; Wagenaar, Hendrik 2003. Deliberative policy analysis. Cambridge: Cambridge University Press.

ICTA et al. (2007). Principles for the oversight of nanotechnologies and nanomaterials. http:// www.etcgroup.org/upload/publication/pdf_file2/principles-for-the-oversight-of-nanotechn ologies-and-nanomaterials.pdf (Zugriff vom 15.12.2011).

IG Metall 2007. »Nanotechnologie: Hope or Hype? «, in Wirtschaft aktuell 14, S. 1-2.

Laclau, Ernesto 1998. »Von den Namen Gottes «, in Das Undarstellbare der Politik. Zur Hegemonietheorie Ernesto Laclaus, hrsg. v. Marchart, Oliver, S. 265-281. Wien: Turia + Kant.

Leviathan, 40. Jg., 1/2012 
Laclau, Ernesto 2005. On populist reason. London, New York: Verso.

Laclau, Ernesto; Mouffe, Chantal 1985. Hegemony and socialist strategy: towards a radical democratic politics. London, New York: Verso.

Lösch, Andreas; Gammel, Stefan; Nordmann, Alfred 2008. „Observieren - Sondieren - Regulieren. Zur gesellschaftlichen Einbettung nanotechnologischer Entwicklungsprozesse ", in Jenseits von Regulierung: Zum politischen Umgang mit der Nanotechnologie, hrsg. v. Gammel, Stefan; Lösch, Andreas; Nordmann, Alfred, S. 16-93. Heidelberg: AKA.

Mitsch, Frank; Mitchell, Jennifer S. 1999. Ag Biotech: Thanks, but no thanks? Deutsche Banc Alex. Brown. http://www.biotech-info.net/Deutsche.pdf (Zugriff vom 15.12.2011).

Mooney, Pat 2006. »Ein technologischer Tsunami kommt auf uns zu «, in politische ökologie 101, S. 37-40.

Mouffe, Chantal 2007. Über das Politische. Wider die kosmopolitische Illusion. Frankfurt a. M.: Suhrkamp.

Nanokommission 2007. Hintergrundpapier zum NanoDialog. http://osha.europa.eu/data/links/ osh_dbcontent.2007-09-13.3743485649 (Zugriff vom 09.01.2012).

NNAP 2008. The National Nanotechnology Initiative: second assessment and recommendations of the National Nanotechnology Advisory Panel. Washington, D.C.: U.S. Government Printing Office.

Poulter, Sean 2008. »Alert over the march of the sgrey goo in nanotechnology Frankenfoods «, in Daily Mail vom 2.1.2008. http://www.dailymail.co.uk/pages/live/articles/technology/techn ology.html?in_page_id=1965\&in_article_id=505561 (Zugriff vom 15.12.2011).

Ross, Philip E. 2006. »Angst vor den Nanogiften ", in Technology Review vom 25.5.2006. http:// www.heise.de/tr/artikel/73533 (Zugriff vom 15.12.2011).

Sachverständigenrat für Umweltfragen 2011. Vorsorgestrategien für Nanomaterialien. Sondergutachten. Berlin: Hausdruck.

SCENIHR 2005. The appropriateness of existing methodologies to assess the potential risks associated with engineered and adventitious products of nanotechnologies. SCENIHR/002/ 05. http://ec.europa.eu/health/ph_risk/committees/04_scenihr/docs/scenihr_o_003.pdf (Zugriff vom 15.12.2011).

Soil Association 2005. Proposed ban on nanotechnology. Information sheet nanotechnology (Version 3). http://92.52.112.178/web/sa/saweb.nsf/848d689047cb466780256a6b002989 80/ebd8481eeee81872802570b5005e192d/\$FILE/Nanotechnology.pdf (Zugriff vom 15.12.2011).

Swiss Re 2004. Nanotechnologie. Kleine Teile - Grosse Zukunft? Zürich: Schweizerische Rückversicherungs-Gesellschaft. http://www.nanotruck.de/fileadmin/user_upload/Berichte\%20u nd\%20Druckschriften/Literaturliste/_SwissRe_Nanorisk_2005.pdf (Zugriff vom 15.12.2011).

Umweltbundesamt 2006. Nanotechnik: Chancen und Risiken für Mensch und Umwelt. http:// www.dialog-nanopartikel.de/Hintergrundpapier\%20nanotechnik_UBA.pdf (Zugriff vom 09.01.2012).

Wörle-Knirsch, Jörg; Krug, Harald F. 2007. »Risikoforschung und toxikologische Bewertung von Nanomaterialien, in Nano. Chancen und Risiken aktueller Technologien, hrsg. v. Gazsó, André; Greßler, Sabine; Schiemer, Fritz, S. 101-114. Wien: Springer-Verlag.

Wullweber, Joscha 2006. »Der Mythos Nanotechnologie. Die Entstehung und Durchsetzung einer neuen Inwertsetzungstechnologie", in Peripherie 101/102, S. 99-118.

Wullweber, Joscha 2008. "Nanotechnology - an empty signifier à venir. A delineation of a techno-socio-economical innovation strategy ", in Science, Technology and Innovation Studies 4, 1, S. 27-45.

Wullweber, Joscha 2010. Hegemonie, Diskurs und Politische Ökonomie. Das Nanotechnologie-Projekt. Baden-Baden: Nomos. 
Zusammenfassung: $\mathrm{Ob}$ sich neue Technologien als Produkte und Anwendungen durchsetzen können, hängt stark von ihrer Akzeptanz in der Gesellschaft ab. Am Beispiel der Nanotechnologie wird aufgezeigt, wie in den letzten Jahren um gesellschaftliche Akzeptanz gerungen wurde. Anhand von diskurs- und hegemonietheoretischen Konzepten werden verschiedene Strategien im Diskurs um die Nanotechnologie identifiziert. Die bislang eher positive Stimmung gegenüber der Nanotechnologie ist demnach kein Zufall, sondern in nicht unbedeutendem Maße auf erfolgreiche Diskursstrategien zurückzuführen.

Stichwörter: Hegemonie, Governance, Nanotechnologie, Diskurs, Strategie

\section{Hegemonic strategies: the struggle for acceptance in the political governance of nanotechnology}

Summary: Whether new technologies can prevail as products and applications strongly depends on their degree of acceptance in society. The struggle for such acceptance is demonstrated in an examination of the recent discourse surrounding nanotechnology. Concepts from the theories of discourse and hegemony are used to identify the various strategies which have been pursued to shape social acceptance. It is no accident that attitudes towards nanotechnology to date have tended to be positive. In no small measure this development is the result of successful discourse strategies.

Keywords: Hegemony, governance, nanotechnology, discourse, strategy

\section{Autor}

Dr. Joscha Wullweber

Universität Kassel

Nora-Platiel-Str. 1

34127 Kassel

joscha.wullweber@uni-kassel.de

Leviathan, 40. Jg., 1/2012 https://doi.org/10.5771/0340-0425-2012-1-4 